\title{
Herd immunity is not a realistic exit strategy during a COVID-19 outbreak
}

Ed Slot ( $\nabla$ e.slot@sanquin.nl )

Sanquin Research

Boris M. Hogema

Sanquin Research

Chantal B.E.M. Reusken

National Institute for Public Health and the Environment

Johan H. Reimerink

National Institute for Public Health and the Environment

Michel Molier

Sanquin Research

Jan H.M. Karregat

Sanquin Research

Johan IJlst

Sanquin Laboratory Services

Věra M.J. Novotný

Sanquin Blood Bank

René A.W. van Lier

University of Amsterdam

Hans L. Zaaijer ( $\sim$ h.zaaijer@sanquin.nl )

Amsterdam UMC

\section{Research Article}

Keywords: coronavirus, COVID-19, exit strategy, herd immunity, pandemic, SARS-CoV-2, seroconversion, seroprevalence, serosurvey, social distancing

Posted Date: April 29th, 2020

DOl: https://doi.org/10.21203/rs.3.rs-25862/v1

License: (a) This work is licensed under a Creative Commons Attribution 4.0 International License. Read Full License 


\section{Abstract}

The world is combating an ongoing COVID-19 pandemic ${ }^{1-4}$. Health-care systems, society and the economy are impacted in an unprecedented way. It is unclear how many people have contracted the causative coronavirus (SARS-CoV-2) unknowingly. Therefore, reported COVID-19 cases do not reflect the true scale of outbreak ${ }^{5-9}$. Natural herd immunity has been suggested as a potential exit strategy during COVID-19 outbreaks, which may arise when $50-67 \%$ of a community has been infected ${ }^{10}$. Here we present the prevalence and distribution of antibodies to SARS-CoV-2 in a healthy adult population of a highly affected country using a novel immunoassay, indicating that one month into the outbreak (i) the seroprevalence in the Netherlands is $2.7 \%$ with substantial regional variation, (ii) the hardest-hit areas show a seroprevalence of up to $9.5 \%$, (iii) the seroprevalence is sex-independent throughout age groups (18-72 years), (iv) antibodies are significantly more often detected in younger people (18-30 years), and (v) the number of immune individuals in the current epidemic stage is far below the herd immunity threshold. This study provides vital information on the extent of virus spread in a country where social distancing is in place, concluding that herd immunity to SARS-CoV-2 is not a realistic short-term exit strategy option.

\section{Introduction}

Coronavirus disease 2019 (COVID-19) is an emerging infectious disease caused by the novel severe acute respiratory syndrome coronavirus 2 (SARS-CoV-2). The disease was first documented in humans in China in December 2019. The virus has since spread globally by person-to-person transmission, resulting in an ongoing pandemic impacting public health, health-care systems, society and the economy across the world ${ }^{1-4}$.

Common clinical COVID-19 manifestations include fever, cough, fatigue, expectoration, shortness of breath, dyspnoea, and muscle soreness ${ }^{11}$. The median incubation period is estimated to be 5.1 days ( $95 \%$ $\mathrm{Cl}, 4.5$ to 5.8 days), and $97.5 \%$ of those who develop symptoms will do so within 11.5 days $(95 \% \mathrm{Cl}, 8.2$ to 15.6 days) of infection ${ }^{12}$. While the majority of patients show only mild or moderate symptoms, some progress to viral pneumonia, acute respiratory distress syndrome (ARDS), systemic inflammatory response syndrome (SIRS), multiple organ failure (MOF), and death.

As of 25 April 2020 more than 2.7 million laboratory-confirmed COVID-19 cases have been reported including 187,705 deaths, affecting 213 countries and territories around the world ${ }^{4}$. In many countries lockdown strategies have been implemented and social distancing is mandatory to reduce person-toperson transmission and thereby protect citizens, mitigate the coronavirus spread and control the associated impact on health-care systems including intensive care capacities, awaiting a significant decrease of virus circulation, population-based immunity through natural exposure to the virus (herd immunity), or the development of effective vaccines or therapeutics ${ }^{13,14}$. 
Herd immunity to SARS-CoV-2 might arise when enough people become infected and recover, developing immunity to the virus in the process ${ }^{15}$. Above the herd immunity threshold (HIT) the infection may no longer persist in the population. The HIT of SARS-CoV-2 is estimated at 50 to $67 \%$, but the incidence of symptomatic and asymptomatic SARS-CoV-2 cases in an epidemic setting is unclear to date ${ }^{10}$.

Assuming that antibodies to SARS-CoV-2 are produced by the immune system in response to virus exposure, serology-based tests for SARS-CoV-2 may be used to discover the true extent of SARS-CoV-2 infections and to monitor the COVID-19 pandemic, but highly accurate immunoassays were not available to date ${ }^{16,17}$. As a result, antibodies to SARS-CoV-2 have not yet been studied on a country level.

High-quality data about immune response kinetics of SARS-CoV-2 and population-based serological studies are urgently needed to understand the extent of the spread of and immunity to the virus. Here we present the prevalence and distribution of antibodies to SARS-CoV-2 under social distancing in a healthy adult population of a highly affected country, one month into the outbreak. Should natural herd immunity be taken into account as a realistic exit strategy option?

\section{Materials And Methods}

\section{Setting}

The Netherlands is the most densely populated country of Europe with 17.4 million human inhabitants (421 per square kilometer). The first Dutch case of COVID-19 was reported on 27 February 2020. An outbreak ensued, culminating in 10,381 hospital admissions, 37,190 laboratory-confirmed COVID-19 cases including 4,409 documented fatalities by 25 April $2020^{18}$. Social distancing and lockdown interventions were implemented nationwide on 15 March 2020.

\section{Subjects and sampling}

We studied plasma and serum samples of 7,361 adult blood plasma donors and 153 convalescent plasma donors from throughout the Netherlands, collected from 1 until 15 April 2020. Donors were enrolled in the study if they were accepted for routine donation including informed consent, without further selection. At the time of donation donors must be completely healthy, but they may have been ill in the past, provided that they recovered at least two weeks before. Hence our study covers potential symptomatic cases of COVID-19 in the first four weeks of the outbreak. Subjects were defined by age (1872 years), gender, and zip code of the subject's residence. All age groups were well represented with a balanced distribution of male and female subjects residing in all regions of the Netherlands.

\section{Serological testing}

Donor samples were screened for antibodies to SARS-CoV-2 using a SARS-CoV-2 total antibody ELISA (Wantai Biological Pharmacy Enterprise Co., Ltd., Beijing, China) following the manufacturer's instructions. Samples testing reactive $(\mathrm{OD} / \mathrm{CO}$ ratio $\geq 1)$ were re-tested and considered positive if the re- 
test was reactive. For the majority of positive donors an archived sample of a previous donation from before the start of the outbreak was available and tested, to confirm seroconversion. Briefly, the Wantai ELISA used is a 'double antigen sandwich assay'. This assay format has the following advantages: the solid phase is coated with recombinant SARS-CoV-2 antigens, which simultaneously bind antibody isotypes (IgA, IgM, IgG) directed to SARS-CoV-2. For detection, not a labeled anti-antibody is used, but again a specific antigen-antibody binding takes place, using labeled recombinant SARS-CoV-2 antigen. Additional testing was performed using a SARS-CoV-2 IgM ELISA (Wantai Biological Pharmacy Enterprise Co., Ltd., Beijing, China) following the manufacturer's instructions.

\section{Validation and evaluation}

Performance characteristics of the Wantai SARS-CoV-2 total antibody ELISA have recently been reported, indicating a sensitivity of $100 \%$ in PCR-confirmed COVID-19 cases (after 10-13 days following the onset of symptoms) and a specificity of $99.1-100 \%{ }^{19,20}$. We additionally validated the ELISA using panels of plasma and serum samples from (i) Dutch blood donors collected in March and April 2018 ( $n=282 ; 1 / 282$ seropositive), (ii) PCR-confirmed COVID-19 patients admitted to Dutch intensive care units in March 2020 ( $n=10 ; 9 / 10$ seropositive; $1 / 10$ seronegative), (iii) Dutch PCR-confirmed COVID-19 patients with only mild symptoms ( $n=11 ; 11 / 11$ seropositive), (iv) Dutch plasma donors with a documented PCR-positive test result who were at least 14 days fully recovered from mild or moderate COVID-19 symptoms ( $n=153$; 151/153 seropositive), and ( $v$ ) patients with common HCoV, CMV or EBV infection ( $n=40 ; 0 / 40$ seropositive).

\section{Statistical analysis}

Association between the prevalence of anti-SARS-CoV-2 antibodies and age, sex and region of residence was assessed by logistic regression analysis using $R$ v.3.5.2.

\section{Ethics statement}

Samples were collected only from voluntary, non-remunerated donors who provided written informed consent as part of routine donor selection and blood collection procedures. The study was reviewed and approved by the Ethics Advisory Council of Sanquin Blood Supply Foundation.

\section{Results}

In total 7,361 donations were tested from donors without known history of COVID-19, of which 230 were repeat reactive in the Wantai total antibody assay (3.1\%). For 218/230 repeat reactive donors archived material of a previous donation was available for testing, showing seroconversion in 188/218 donors (86\%) and pre-outbreak reactivity in 30/218 (14\%); for 12 repeat reactive donors no pre-outbreak samples were available. Positive IgM test results (in subsets of donors) were found as follows: in 144/180 (80\%) seroconverters ( 8 donors not tested); in $3 / 28$ (11\%) donors with pre-outbreak reactivity ( 2 donors not tested); and in 8/12 (67\%) donors without a previous donation. Based on demonstrated seroconversion, 
188/7,361 (2.6\%) donors experienced recent SARS-CoV-2 infection. If donors without a previous donation are included this number is $200 / 7,361$ (2.7\%). The resulting positive predictive value of the test is $86 \%$ with a specificity of $99.6 \%$.

Figure 1 shows the distribution of ELISA signals in the 230 antibody positive donors, subdivided in seroconverting donors (in red), donors with pre-outbreak reactivity (in white) and donors without a previous donation (in blue). IgM reactivity is depicted using colored dots for each donor. The 188 donors with confirmed seroconversion and the twelve donors from whom no archive sample was available were considered seropositive in the subsequent analyses.

The prevalence of antibodies to SARS-CoV- 2 was not different for men and women (2.70 vs $2.73 \%$ ). Logistic regression and chi-square analysis showed that the seroprevalence in donors aged 18-30 years was significantly higher than in other age groups (Table $1 ; p=0.026$ ).

The seroprevalence varied by geographic region. Figure 2 shows the distribution for the 26 municipal health service regions of the Netherlands, demonstrating a north to south gradient.

Table 1 Prevalence of anti-SARS-CoV-2 antibodies in different age groups; 1-15 April 2020

\begin{tabular}{|c|c|c|c|}
\hline Age group & \multicolumn{2}{|c|}{ Anti-SARS-CoV-2 prevalence } & 95\% confidence interval \\
\hline & $52 / 1,251$ & $(4.2 \%)$ & $3.1-5.4 \%$ \\
\hline $18-30$ & & & \\
\hline $31-40$ & $22 / 882$ & $(2.5 \%)$ & $1.6-3.8 \%$ \\
\hline $41-50$ & $31 / 1,354$ & $(2.3 \%)$ & $1.6-3.2 \%$ \\
\hline $51-60$ & $48 / 2,132$ & $(2.3 \%)$ & $1.7-3.0 \%$ \\
\hline $60-72$ & $47 / 1,742$ & $(2.7 \%)$ & $2.0-3.8 \%$ \\
\hline
\end{tabular}

\section{Discussion}

The world is facing unprecedented challenges with communities and economies everywhere affected by the COVID-19 pandemic. Social distancing and other public health measures are placing an increasing psychological burden on people, resulting in an increasing need for exit strategies ${ }^{21}$.

As the situation evolves, public health interventions significantly lower the real-time reproduction number $\left(R_{t}\right)$, which estimates the average number of secondary cases one case produces in a population made up of both susceptible and non-susceptible hosts ${ }^{22}$. If person-to-person transmission is limited and the $R_{t}$ drops, the pandemic may be controlled on a population level until an effective SARS-CoV-2 vaccine or pharmacological therapy for the treatment of COVID-19 becomes available ${ }^{13,14}$.

Another way out may be acquiring natural herd immunity to SARS-CoV-2 as an indirect protection conferred by immune individuals to the susceptible ones in the population. If herd immunity to SARSCoV-2 is attainable during the COVID-19 pandemic, policy makers may take this into account as a realistic exit strategy option. The $2.7 \%$ seroprevalence found in our study clearly shows that the number of putative immune individuals in the current epidemic stage under social distancing is far below the 50- 
$67 \%$ HIT. Without social distancing the chains of infection will resume shortly, even in the hardest-hit areas with a seroprevalence of up to $9.5 \%$. We anticipate this goes not only for the Netherlands, but for all countries affected by the pandemic where social distancing is in place.

Our study further indicates that the prevalence of antibodies to SARS-CoV-2 varies substantially among areas in a highly affected country and is sex-independent throughout age groups (18-72 years) in the healthy population. Noteworthy, antibodies are significantly more often detected in younger people (18-30 years), which might be related to different social behaviours and a higher exposure to the virus before social distancing was implemented. Of course, the study only covers SARS-CoV- 2 infections in the first weeks of the outbreak and the results cannot be extrapolated to children, non-healthy adults and elderly aged $>72$ years. However, we believe that the prevalence of antibodies to SARS-CoV-2 found in a sample of the Dutch donor population provides an important first indication of the general seroprevalence until more specific data become available.

The Wantai SARS-CoV-2 total antibody ELISA shows performance characteristics superior to other immunoassays currently on the market ${ }^{19,20}$. However, despite the high specificity of the Wantai ELISA (99.6\%), $14 \%$ of seroreactive individuals in our study were false positive based on archive samples collected prior to the COVID-19 outbreak. The positive predictive value (PPV) of the test was calculated as $99 \%, 88 \%$, and $72 \%$ in areas with a seroprevalence of $4-10 \%, 2-4 \%$, and $<2 \%$, respectively. Notably, the PPV increases with the seroprevalence and hence will increase as the outbreak proceeds. This phenomenon must be taken into account if SARS-CoV-2 antibody testing would in the future be considered for diagnostic services and for reducing social distancing measures.

It is unknown whether the presence of SARS-CoV-2 antibodies reflects immunity, be it short term or long term $^{23,24}$. Wu and colleagues studied the levels and time course of neutralizing antibodies (nAbs) in 175 patients who experienced mild COVID-19 symptoms ${ }^{25}$. In those patients nAbs were detected from day 1015 after onset of disease and remained thereafter. Interestingly, middle-aged and elderly patients had significantly higher $n A b$ titers $(P<0.0001)$ than younger patients. Ten patients had undetectable $n A b$ titers (ID50: <40) and two showed very high titers (ID50: 15,989 and 21,567). These findings indicate that antibodies to SARS-CoV-2 in asymptomatic individuals and in patients with only mild symptoms may have limited neutralizing capacity. Like in other infections non-nAbs may play a significant role in decreasing the viral load, e.g. via Fc receptor-mediated uptake in innate cells, leading to partial or even total protection ${ }^{26}$. Therefore SARS-CoV-2 antibody-mediated immunity, including the role and formation of nAbs and non-nAbs, needs further research both to ascertain whether asymptomatic and non-severe cases become immune to the virus and to assess the relevance of SARS-CoV-2-specific antibodies for the development of safe and effective (i) vaccines, (ii) treatment of COVID-19 patients with convalescent plasma transfusions, and (iii) plasma-derived medicinal products (anti-SARS-CoV-2 hyperimmune globulins) ${ }^{27-29}$.

Gilbert and colleagues have proposed an exit strategy approach in which at first only seropositive individuals, recovered from SARS-CoV-2 infection, return to their normal lives ${ }^{30}$. When the pandemic 
subsides, gradually younger low-risk people without symptoms might follow. Such an approach would slowly build up immunity in the population, mitigating the impact on health-care systems and intensive care capacities and reducing the intensity of future waves of the pandemic. This would make it possible to reconcile the advantages of two opposing strategies that have been proposed: the strategy of global containment of the population, which is economically and socially costly, and the strategy based on natural herd immunity, which potentially involves a substantial human cost if done too fast. To put this approach in context, we stress that serology-based tests in general come with important pitfalls until accurate confirmatory testing is available and that the protective effect of SARS-CoV-2-specific antibodies is not yet known. An antibody testing-based exit strategy could thus lead to a resurgence of community spread of the virus.

In conclusion, natural herd immunity to SARS-CoV-2 under social distancing is far away in the current epidemic stage and therefore is not a realistic short-term exit strategy option. As a consequence, downscaling public health interventions in the short term may significantly increase the $R_{t}$ and fuel the COVID-19 pandemic, resulting in potentially uncontrollable situations. It is pivotal to continuously revisit public health and exit strategies based on updated data about the extent of the spread of and immunity to the virus on the population level.

\section{Declarations}

\section{ACKNOWLEDGMENTS}

We thank Femmeke Prinsze for data extraction from the Sanquin donor database and Marielle van Rijswijk for help with retrieving archived samples.

\section{AUTHOR CONTRIBUTIONS}

$\mathrm{ES}, \mathrm{HZ}, \mathrm{BH}, \mathrm{CR}$ and JR conceived the study, designed the experiments and analyzed the data. MM, JK and $\mathrm{JI}$ processed the samples, performed the experiments and collected data. $E S, H Z, B H, R L, C R, J R, V N, M M$, $\mathrm{JK}$ and $\mathrm{JI}$ wrote the paper (in order of contribution).

\section{FUNDING}

The study has been funded by Sanquin Blood Supply Foundation and the Dutch Ministry of Health, Welfare and Sport. The funder had no role in study design, data collection and analysis, decision to publish, or preparation of the manuscript.

\section{COMPETING INTERESTS}

The authors declare no competing interests.

\section{DATA AVAILABILITY STATEMENT}


All relevant data are within the paper.

\section{References}

1. Wu, F., Zhao. S., Yu, B., Chen, Y.M., Wang, W., et al. A new coronavirus associated with human respiratory disease in China. Nature 579, 265-269 (2020).

2. Zheng, J. SARS-CoV-2: an Emerging Coronavirus that Causes a Global Threat. Int J Biol Sci. 16, 1678-1685 (2020).

3. Steffens, I. A hundred days into the coronavirus disease (COVID-19) pandemic. Euro Surveill. 25, pii=2000550 (2020).

4. World Health Organization. Coronavirus disease (COVID-2019) situation report. Accessed on 25 April 2020: https://www.who.int/emergencies/diseases/novel-coronavirus-2019/situation-reports/ (2020).

5. Li, R., Pei, S., Chen, B., Song, Y., Zhang, T., et al. Substantial undocumented infection facilitates the rapid dissemination of novel coronavirus (SARS-CoV2). Science. Preprint at doi: 10.1126/science.abb3221 (2020).

6. Lipsitch, M., Swerdlow, D.L., Finelli, L. Defining the Epidemiology of Covid-19 - Studies Needed. N Engl J Med. 382, 1194-1196 (2020).

7. Park, M., Cook, A.R., Lim, J.T., Sun, Y., Dickens, B.L. A Systematic Review of COVID-19 Epidemiology Based on Current Evidence. J Clin Med. 9, 967 (2020).

8. Day, M. Covid-19: four fifths of cases are asymptomatic, China figures indicate. BMJ. Preprint at doi: 10.1136/bmj.m1375 (2020).

9. Adam, D. Special report: The simulations driving the world's response to COVID-19. Nature 580, 316318 (2020).

10. Syal, K. COVID-19: Herd Immunity and Convalescent Plasma Transfer Therapy. J Med Virol. Preprint at doi: 10.1002/jmv.25870 (2020).

11. Zhu, J., Ji, P., Pang, J., Zhong, Z., Li, H., et al. Clinical characteristics of 3,062 COVID-19 patients: a meta-analysis. J Med Virol. Preprint at doi: 10.1002/jmv.25884 (2020).

12. Lauer, S.A., Grantz, K.H., Bi, Q., Jones, F.K., Zheng, Q., et al. The Incubation Period of Coronavirus Disease 2019 (COVID-19) From Publicly Reported Confirmed Cases: Estimation and Application. Ann Intern Med. Preprint at doi: 10.7326/M20-0504 (2020).

13. Sanders, J.M., Monogue, M.L., Jodlowski, T.Z., Cutrell J.B. Pharmacologic Treatments for Coronavirus Disease 2019 (COVID-19): A Review. Preprint at doi: 10.1001/jama.2020.6019.

14. Tu, Y.F., Chien, C.S., Yarmishyn, A.A., Lin, Y.Y., Luo, Y.H., et al. A Review of SARS-CoV-2 and the Ongoing Clinical Trials. Int J Mol Sci. 21, doi: 10.3390/ijms21072657 (2020).

15. Fine, P., Eames, K., Heymann, D.L. "Herd immunity": a rough guide. Clin Infect Dis. 52, 911-916 (2011).

16. Subbaraman, N. Coronavirus tests: researchers chase new diagnostics to fight the pandemic. Nature. Preprint at doi: 10.1038/d41586-020-00827-6 (2020). 
17. Petherick, A. Developing antibody tests for SARS-CoV-2. Lancet 395, 1101-1102 (2020).

18. National Institute for Public Health and the Environment (RIVM). Accessed on 25 April 2020: https://www.rivm.nl/documenten/epidemiologische-situatie-covid-19-in-nederland-25-april-2020 (2020).

19. Zhao, J., Yuan, Q., Wang, H., Liu, W., Liao, X., et al. Antibody responses to SARS-CoV-2 in patients of novel coronavirus disease 2019. Clin Infect Dis. Preprint at doi: 10.1093/cid/ciaa344 (2020).

20. Lassaunière, R., Frische, A., Harboe, Z.B., Nielsen, A.C.Y., Fomsgaard, A., et al. Evaluation of nine commercial SARS-CoV-2 immunoassays. Preprint at https://doi.org/10.1101/2020.04.09.20056325 (2020).

21. Fiorillo, A., Gorwood, P. The consequences of the COVID-19 pandemic on mental health and implications for clinical practice. Eur Psychiatry. Preprint at doi: 10.1192/j.eurpsy.2020.35 (2020).

22. Yuan, J., Li, M., Lv, G., Lu, Z.K. Monitoring Transmissibility and Mortality of COVID-19 in Europe. Int J Infect Dis. Preprint at doi: 10.1016/j.ijid.2020.03.050 (2020).

23. Okba, N.M.A., Müller, M.A., Li, W., Wang, C., GeurtsvanKessel, C.H., et al. Severe Acute Respiratory Syndrome Coronavirus 2-Specific Antibody Responses in Coronavirus Disease 2019 Patients. Emerg Infect Dis. Preprint at doi: 10.3201/eid2607.200841 (2020).

24. Huang, A.T., Garcia-Carreras, B., Hitchings, M.D.T., Yang, B., Katzelnick, L.C., et al. A systematic review of antibody mediated immunity to coronaviruses: antibody kinetics, correlates of protection, and association of antibody responses with severity of disease. Preprint at https://doi.org/ doi.org/10.1101/2020.04.14.20065771 (2020).

25. Wu, F., Wang, A., Liu, M., Wang, Q., Chen, J., et al. Neutralizing antibody responses to SARS-CoV-2 in a COVID-19 recovered patient cohort and their implications. Preprint at https://doi.org/10.1101/2020.03.30.20047365 (2020).

26. Ilinykh, P.A., Huang, K., Santos, R.I., Gilchuk, P., Gunn, B.M., et al. Non-neutralizing Antibodies from a Marburg Infection Survivor Mediate Protection by Fc-Effector Functions and by Enhancing Efficacy of Other Antibodies. Cell Host Microbe. Preprint at https://doi.org/10.1016/j.chom.2020.03.025 (2020).

27. Roback, J.D., Guarner, J. Convalescent Plasma to Treat COVID-19: Possibilities and Challenges. JAMA. Preprint at doi: 10.1001/jama.2020.4940 (2020).

28. Chen, L., Xiong, J., Bao, L., Shi, Y. Convalescent plasma as a potential therapy for COVID-19. Lancet Infect Dis. 20, 398-400 (2020).

29. CoVIg-19 Plasma Alliance. Donate your plasma to help develop a potential treatment for COVID-19. Accessed on 25 April 2020: https://www.covig-19plasmaalliance.org/en-us\#info (2020).

30. Gilbert, M., Dewatripont, M., Muraille, E., Platteau, J-P., Goldman, M. Preparing for a responsible lockdown exit strategy. Nat Med. Preprint at https://doi.org/10.1038/s41591-020-0871-y (2020).

\section{Figures}




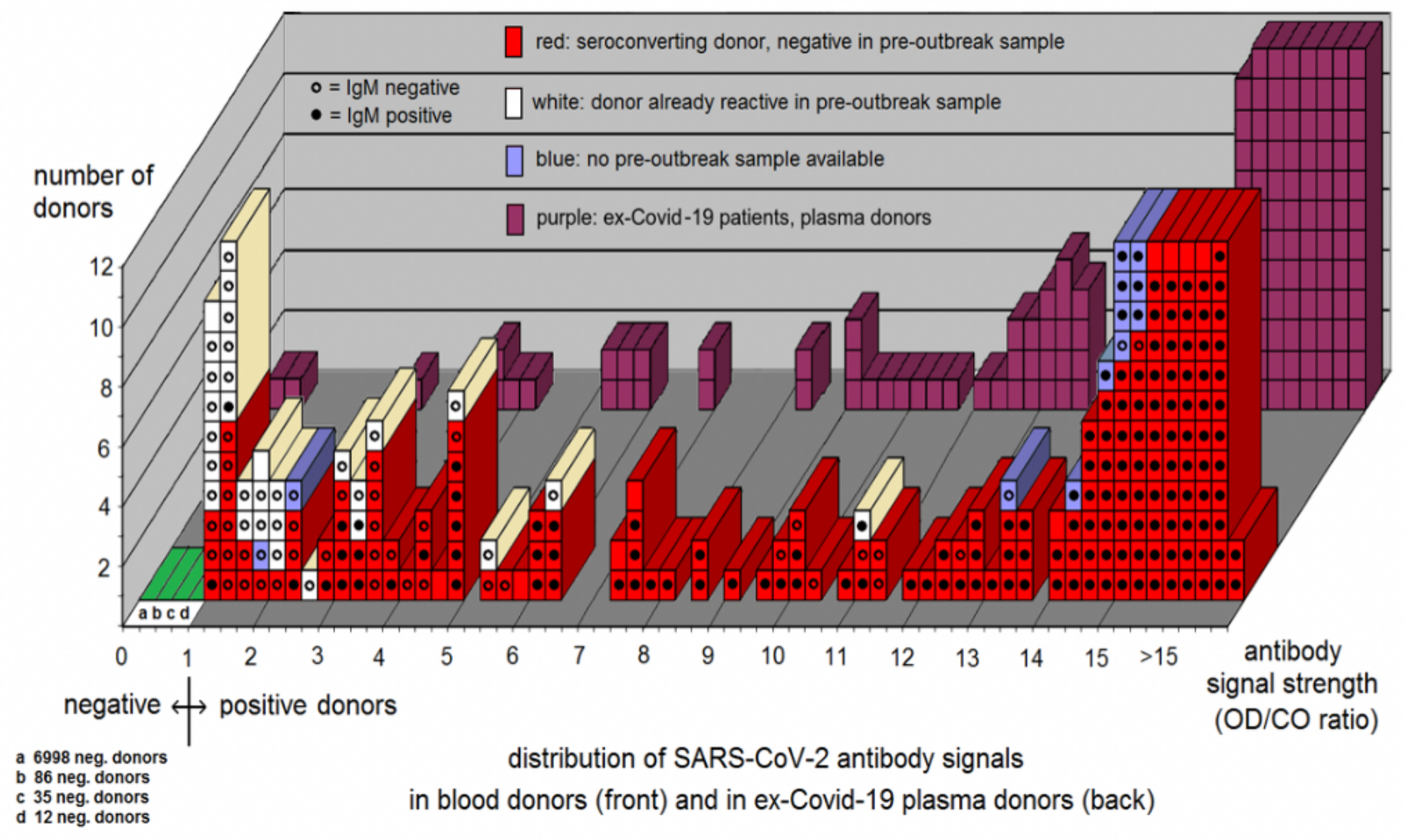

Figure 1

Distribution of SARS-CoV-2 antibody signals; 1-15 April 2020 


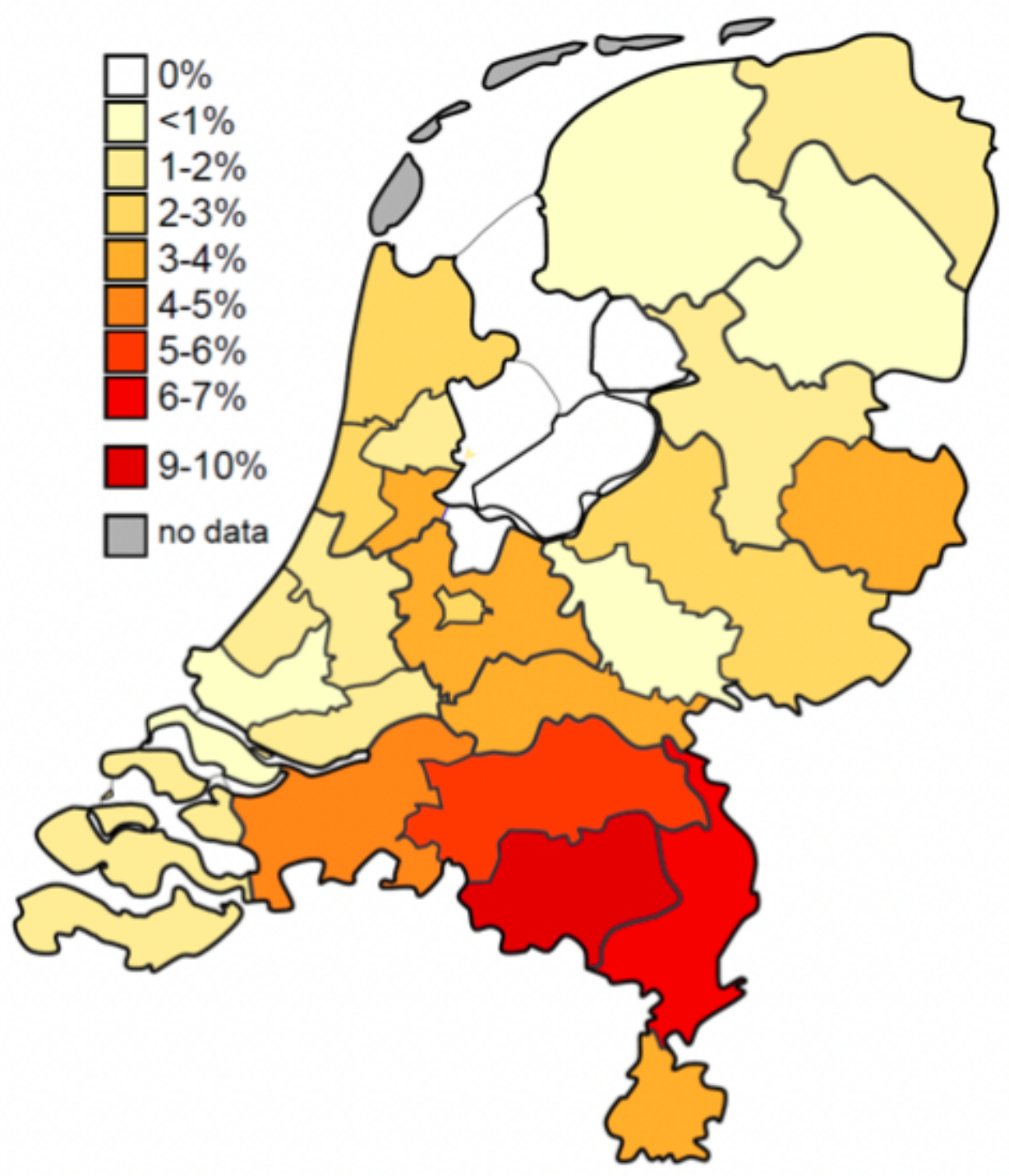

Figure 2

SARS-CoV-2 seroprevalence in the Dutch blood donor population; 1-15 April 2020 